\title{
Frecuencia de Macrosomía Neonatal en mujeres embarazadas con Diabetes Gestacional en el Hospital Vicente Corral Moscoso. Cuenca Ecuador, en el periodo 2009-2014
}

Frequency of Neonatal macrosomia in pregnant women with gestational Diabetes at the Vicente Corral Moscoso Hospital. Cuenca Ecuador, In the period 2009-2014

Juana Karina Cobos. ${ }^{1}$, Juan Enrique Rodas Mosquera. ${ }^{2} \&$ Johana Carolina Carrión Crespo. $^{3}$

\begin{abstract}
.
\footnotetext{
1 Médico en libre ejercicio, Riobamba, Ecuador, juanic1992@hotmail.com

2 Médico en libre ejercicio, Riobamba, Ecuador, juanen90@ hotmail.com

3 MSP-Distrito 01D02, Riobamba, Ecuador, karocarrion@gmail.com
}

DOI: https://doi.org/10.33262/cienciadigital.v9i2.425

The objective of this study is to determine the frequency of neonatal macrosomia in pregnant women with gestational diabetes at the Vicente Corral Moscoso's Hospital in period 2009-2014. This is a quantitative, descriptive and retrospective research based on a review of 106 medical records and tabulated in Microsoft Excel. 2010, frequencies and percentages were obtained and simple tables and graphs were elaborated.

The results showed that the predominant maternal age was more than 30 years (39\%), in gestational age $58 \%$ was found between the 42 or more weeks, $75 \%$ of the products had a tendency to macrosomia with values equal to or more than 3999 grams at birth, in neonatal sex between men and women a percentage of $53 \%$ and $47 \%$ respectively was found, there were 90 vaginal births $(85 \%)$ and 16 caesarean $(15 \%)$, according to the maternal weight, $30 \%$ was found in the group of 81 to $90 \mathrm{Kg}$ and $13 \%$ in more than 
91kg. Conclusion: Three quarters of pregnant women with Gestational Diabetes in the study group had fetal macrosomia products.

Keywords: neonatal macrosomia, gestational diabetes, pregnant women

\section{Resumen.}

El objetivo de esta investigación consideró determinar la frecuencia de macrosomía neonatal en mujeres embarazadas con diagnóstico de Diabetes Gestacional en el Hospital Vicente Corral Moscoso en el periodo 2009-2014. El tipo de estudio fue cuantitativo, descriptivo y retrospectivo realizado en base a revisión de 106 historias clínicas, se procesó la información con Microsoft Excel 2010, se obtuvieron frecuencias y porcentajes y se elaboraron tablas simples y gráficos. Los resultados evidenciaron que la edad materna predominante fue mayor de 30 años (39\%), en edad gestacional el $58 \%$ se encontró entre las 42 o más semanas, el $75 \%$ de los productos presentaron tendencia a la macrosomía con valores iguales o mayores a 3999 gramos al nacer, en el sexo neonatal entre hombres y mujeres se encontró un porcentaje de 53\% y $47 \%$ respectivamente, hubieron 90 partos vaginales $(85 \%)$ y 16 cesáreas (15\%) según el peso materno el $30 \%$ se encontró en el grupo de 81 a $90 \mathrm{Kg}$ y el 13\% en más de 91 Kg Conclusión: Las tres cuartas partes de embarazadas con Diabetes Gestacional del grupo de estudio tuvieron productos con macrosomía fetal.

Palabras clave: macrosomía fetal, diabetes gestacional, mujeres embarazadas

\section{Introducción.}

El término macrosomía deriva del griego macros (grande) y soma (cuerpo). Tradicionalmente, la macrosomía ha sido definida por el peso al nacimiento, cuando este es mayor a 4000g o por el peso al nacimiento en relación con la edad gestacional (>percentil 90) en base al incrementado riesgo perinatal que presenta (1). Se ha estandarizado que un neonato macrosómico es aquel cuyo peso supera los $3999 \mathrm{~g}$, pero algunos autores proponen un peso superior a $4100 \mathrm{~g}$ o incluso a $4500 \mathrm{~g}$ (2), se concluyó que deben ser motivo de estudio los recién nacidos de menos de 4000g (3999g) pero por arriba del P90 en su edad gestacional e independientemente del sexo (3). 
La Organización Mundial de la Salud (OMS) define a la diabetes gestacional (DG) como "la intolerancia a los carbohidratos resultando en hiperglicemia de gravedad variable que se inicia o se reconoce por primera vez durante el embarazo y alcanza valores que, pese a ser superiores a los normales, son inferiores a los establecidos para diagnosticar una diabetes". En donde existe una alteración en el metabolismo de los hidratos de carbono y debido a esto una insuficiente adaptación a la insulina resistencia causada por los efectos bloqueadores de otras hormonas, como lo es la elevación de la hormona lactógeno placentaria y el cortisol materno, sobre la insulina ya producida ocasionando la condición anteriormente señalada denominada resistencia a la insulina, dichas hormonas comienzan a elevarse a partir de la séptima semana y llegan a su máxima expresión en el tercer trimestre (4). La DG ha ido aumentando su incidencia de la misma manera que la DM tipo 2, y significa un riesgo tanto para la madre como para el hijo, a corto y largo plazo y su prevención, su frecuencia como su diagnóstico y tratamiento precoces son esenciales para mejorar el pronóstico del binomio madre-hijo (5).

La asociación entre diabetes mellitus materna y recién nacido macrosómico es de vital interés en este estudio, y fue reportada por primera vez por Allen en 1939, y Farquhar en 1959, publica la clásica descripción fenotípica del hijo de madre diabética: "rollizo", con facies abultada y "mofletuda", panículo adiposo aumentado y aspecto "pletórico" (6). En la actualidad se conoce con certeza que el estado hipoglucémico fetal, (el mismo que es provocado por la diabetes materna), puede exacerbar el proceso expansivo insular, de esta manera se explica el aumento de tamaño del páncreas en el feto, el mismo que tiene una masa de islotes pancreáticos que constituye el $10 \%$ del total de la masa pancreática (7). Se señala que el exceso de insulina también afecta el crecimiento fetal por dos mecanismos, en primer lugar induce a un aumento de los procesos anabólicos, es decir incrementa la utilización celular de glucosa y también su depósito intracelular en forma de glucógeno en el hígado y el musculo esquelético; propicia la incorporación de los aminoácidos a las proteínas, así como la síntesis de estas, además disminuye el catabolismo proteico y la lipolisis; y en segundo lugar la insulina actúa como factor de crecimiento y provoca tanto hipertrofia como hiperplasia de los tejidos sensibles a su acción, como lo son el hígado, musculo esquelético, corazón, bazo, timo y glándulas suprarrenales, lo que a su vez se traduce en un aumento exagerado del tamaño fetal (8). Durante las últimas semanas de gestación, los hijos de madres 
diabéticas (HMD) depositan 50 al 60\% más de grasa, en comparación con fetos de gestantes no diabéticas, recalcando que en estos fetos el diámetro biparietal (BP) corresponde al rango normal, mientras que la circunferencia abdominal aumenta paulatinamente desde el $7 \mathrm{mo}$ mes de gestación (feto asimétrico), confirmando así que solo hay crecimiento de los tejidos sensibles a la insulina (9).

La O.M.S. propone que se utilicen en la mujer embarazada los mismos procedimientos de diagnóstico de diabetes mellitus que en el resto de las personas, y que toda mujer que reúna los criterios diagnósticos de intolerancia a la glucosa o diabetes mellitus sea considerada y manejada como diabetes mellitus gestacional; mientras que la A.D.A. mantiene los criterios de O’Sullivan y Mahan (10).

La DG no tiene síntomas ni signos propios, sólo complicaciones, es por ello que se recomienda realizar un estudio a las gestantes entre las semanas 24 y 28 (11). Se analizan los exámenes complementarios, entre ellos el tamizaje con una prueba de glucosa en sangre donde se determinará el riesgo, siendo alto cuando el valor es superior a 126mg/dL indicando diabetes preexistente, entre 92-126mg/dL diabetes gestacional y si este es menor a 92mg/dL se realizará una prueba de tolerancia oral de glucosa (PTOG), se indica que a la hora de la ingesta el valor de glucosa será de $180 \mathrm{mg} / \mathrm{dL}$, a las 2 horas $153 \mathrm{mg} / \mathrm{dL}$ con un valor basal menor a $92 \mathrm{mg} / \mathrm{dL}$, si uno o más de estos se altera, se considera DG (12).

El ultrasonido es el "Gold Estándar" para su diagnóstico, este método busca no solo la valoración del peso fetal, sino también la vigilancia durante el tercer trimestre a fin de prevenir problemas como asfixia fetal y optimizar el crecimiento. Esta entidad clínica supone un incremento significativo de la morbilidad materno-fetal (13), con una sensibilidad de un $21.6 \%$ y una especificidad de $98.6 \%$ (14). Existen dos técnicas, la primera es que desde las 29 a 33 semanas se puede determinar el valor de la circunferencia abdominal fetal, y este deberá encontrarse por encima del percentil 70, de esta forma podría establecerse la presencia o no de riesgo de tener hijos macrosómicos (15); la segunda hace relación a la medición del perímetro abdominal a partir de las 37 semanas, en donde valores superiores o iguales a 1,2cm predice dicha patología (16).

Por todo lo señalado anteriormente, la macrosomía debe ser diagnosticada a tiempo ya que es aquella patología más frecuente que desarrollan los HMD, en múltiples estudios realizados 
entre ellos el observado en la revista chilena de ginecología y obstetricia que abarcó a 3981 neonatos, se puede visualizar que los 35 años es la edad materna que presentan la mayoría de gestantes, encontrándose este valor en el límite de este estudio (17). Otro punto a considerar es el peso materno, ya que un IMC mayor a 25 presupone mayor incidencia de desarrollar niños macrosómicos así como realizarse los exámenes oportunos, ya que de no hacerlo, llevará a una gran morbilidad neonatal y por supuesto a dificultades intraparto como partos operatorios con empleo de fórceps, traumatismos en el canal del parto y desproporción cefalopélvica, cesáreas emergentes o hemorragia postparto, haciendo que su manejo y tratamiento se tornen aún más dificultosos y comprometa la salud del binomio madre-hijo (18).

\section{Desarrollo}

La salud materna y perinatal es un proceso esencial para la salud reproductiva, cuyo objeto es garantizar a las mujeres una maternidad saludable y sin riesgos que permita contribuir a la disminución de la morbi-mortalidad materno-fetal. La macrosomía fetal es la complicación más característica llegando a alcanzar porcentajes que superan el $10 \%$ en mujeres embarazadas mayores de 25 años diagnosticadas de diabetes gestacional ya sea por niveles de glucosa mayores a $126 \mathrm{mg} / \mathrm{dl}$ o por una curva de tolerancia a la glucosa compatible con la patología que tratamos. A nivel mundial la prevalencia de la DG se encuentra entre un 2 a 9\%; por otro lado, en México dicho valor corresponde al 4,3\% principalmente en poblaciones que tiene derecho al seguro social, y hasta un $11 \%$ en otras poblaciones (19).

Los fetos macrosómicos son un problema en la sala de partos debido a la posibilidad de complicaciones tanto maternas y fetales, siendo más complejas las últimas. Se relaciona íntimamente con la cobertura y calidad de los servicios de salud prestados y encontramos que en el Ecuador la población rural no tiene total acceso a estos. Este problema no solo radica en la escasa información que se tiene, sino también en la despreocupación o poca importancia de la gestante llevando a un bajo seguimiento del embarazo por parte de un profesional de salud (19).

En el Ecuador la diabetes ocupa el sexto lugar entre las enfermedades crónico degenerativas, encontrándose también la hipertensión arterial y la obesidad entre las principales patologías, alcanzando niveles del 6 al 9\% de la población general, y algunos estudios sugieren que este 
valor alcanza el 12\%; por lo que se recomienda que se lleve a cabo durante el embarazo un adecuado control metabólico y se sugiere a todas las mujeres gestantes realizarse una prueba de O'Sullivan entre las semanas 24-28 del embarazo, para de esta manera detectar cualquier afección (20).

\section{Importancia del problema}

Esta investigación es útil porque suministra información y datos que no se disponían con anterioridad, en este caso acerca de la frecuencia de macrosomía fetal con respecto a las embarazadas con diagnóstico de diabetes gestacional, para el Hospital Vicente Corral Moscoso (HVCM); recordando que esta patología está vinculada con la mayor frecuencia de complicaciones obstétricas, y con el aumento de la incidencia de traumatismos fetales y maternos que la acompañan en el momento del parto y por ende son de gran importancia su estudio. La utilidad del mismo será para que el HVCM pueda analizar los distintos casos y de esta forma pueda asesorar, de la mejor manera a las madres y familias que cursan con esta patología, así como las alternativas para prevención o en el caso el tratamiento óptimo y oportuno para la madre y el feto.

El Hospital Vicente Corral Moscoso y todo su personal médico serán los principales beneficiarios, ya que podrán tomar las medidas respectivas y las decisiones adecuadas con respecto al manejo, ya que verán la realidad en la que se encuentran y de esta forma ayudarían a mejorar la esperanza de vida de las gestantes y sus productos.

\section{Metodología}

Esta investigación fue de tipo cuantitativo, descriptivo, retrospectivo, se realizó en el Hospital Vicente Corral Moscoso, ubicado en la ciudad de Cuenca, Azuay - Ecuador. El universo del estudio fueron las historias clínicas de las madres con diabetes gestacional que llegaron en trabajo de parto al Hospital Vicente Corral Moscoso en el periodo entre el 1 de enero del 2009 al 31 de diciembre del 2014, y la muestra fueron un total de 106 casos. El método empleado fue observacional, la técnica empleada fuere revisión de historias clínicas y para el registro de datos se utilizó un formulario en el que constaban las variables de estudio: edad materna, edad gestacional, la presencia o ausencia de DG durante el embarazo, peso materno, tipo de parto y peso del recién nacido. 
Para el procesamiento de la información se utilizó Microsoft Excel 2010, se sistematizó la información, como estadísticos se utilizaron frecuencias y porcentajes, se elaboraron tablas simples y gráficos, y se analizó con estadística descriptiva.

\section{Resultados}

De los ciento 106 casos analizados, en relación a la edad se observó que cerca del $40 \%$ son mayores de 30 años, y el 16\% en el grupo de 15 a 20 años (Tabla 1), según sobrepeso materno el 30\% se encontró en el grupo de 81 a $90 \mathrm{Kg}$ y el 13\% en más de $91 \mathrm{Kg}$ (Tabla 2), la media marca un valor de $78 \mathrm{~kg}$ que coincide con el mayor porcentaje de casos, la macrosomía se presentó en un 75\% (Tabla 3), la edad gestacional predominante fue entre las 42 o más semanas con un $58 \%$ y una media de 37 . Con respecto a la variable de sexo neonatal el $53 \%$ fue masculino frente al $47 \%$ femenino. En relación al tipo de parto se encontró que el parto normal se presentó con mayor frecuencia (85\%), siendo la minoría la cesárea (15\%). (Figura $\left.\mathrm{N}^{\mathrm{o}} 1\right)$

Tabla 1. Edad materna de mujeres embarazadas con diagnóstico de diabetes gestacional y productos macrosómicos del Hospital Vicente Corral Moscoso periodo 2009-2014,

Cuenca 2019

\begin{tabular}{lll}
\hline \multicolumn{1}{c}{ Edad materna } & Frecuencia & \% \\
\hline $15-20$ & 17 & 16 \\
$21-25$ & 19 & 18 \\
$26-30$ & 29 & 27 \\
Mas 30 & 41 & 39 \\
Total & 106 & 100
\end{tabular}

\section{Fuente: Historias clínicas HVCM}

* La media de edad materna se ubicó en 26 con un desvío estándar de 4,4.

Tabla 2. Mujeres embarazadas con diagnóstico de diabetes gestacional y productos macrosómicos del Hospital Vicente Corral Moscoso periodo 2009-2014 según peso materno, Cuenca 2019 
Peso (kg)

Frecuencia

$\%$

$60-70$

26

25

$71-80$

34

32

$81-90$

32

30

Mas 91

14

13

Total

106

100

DS: 9,1

Fuente: Historias clínicas HVCM

Tabla 3. Mujeres embarazadas con diagnóstico de diabetes gestacional y productos macrosómicos del Hospital Vicente Corral Moscoso periodo 2009-2014, según macrosomía, Cuenca 2019

\begin{tabular}{lll}
\hline Peso $(\mathbf{g})$ & Frecuencia & $\%$ \\
\hline Menos 3999 & 26 & 25 \\
Igual o más a 3999 & 80 & 75 \\
Total & 106 & 100 \\
\hline
\end{tabular}

Media: 3065

DS: 522,91

Fuente: Historias clínicas HVCM

Tabla 4. Edad gestacional de mujeres embarazadas con diagnóstico de diabetes gestacional y productos macrosómicos del Hospital Vicente Corral Moscoso periodo 2009-2014, Cuenca 2019

\begin{tabular}{lll}
\hline Edad gestacional & Frecuencia & $\%$ \\
\hline Menos 37 & 20 & 19 \\
$37-41$ & 25 & 24 \\
42 o mas & 61 & 58 \\
Total & 106 & 100
\end{tabular}

DS: 4 
Fuente: Historias clínicas HVCM

Figura $N^{\circ} 1$

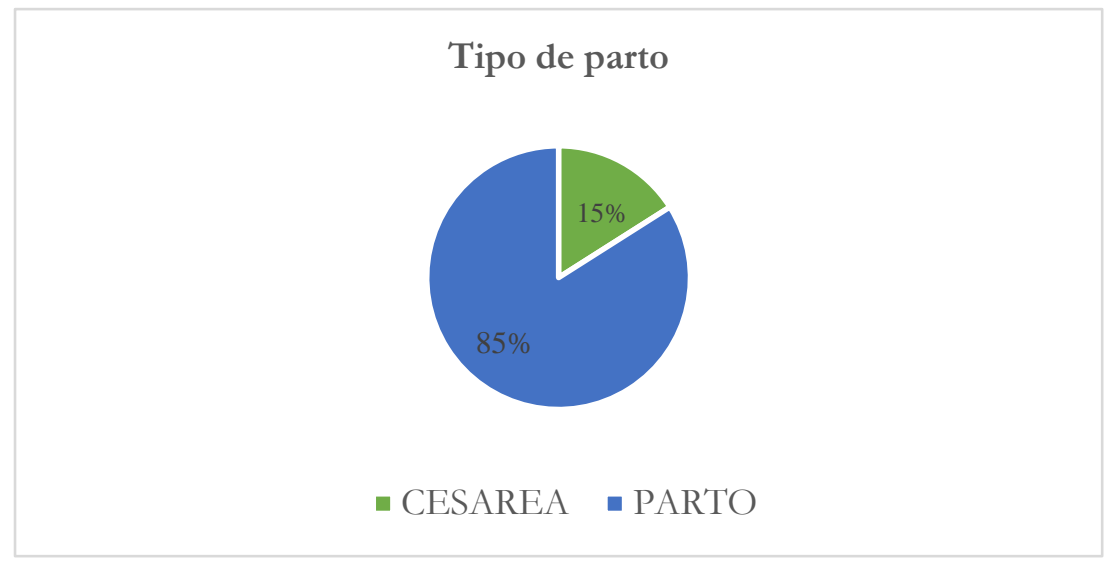

Fuente: Historias clínicas HVCM

\section{Discusión}

El presente estudio tuvo como objetivo determinar la frecuencia de macrosomía fetal presente en productos de embarazadas con diagnóstico de diabetes gestacional en el Hospital Vicente Corral Moscoso en el periodo 2009-201, la frecuencia de diabetes gestacional fue de 106 casos, esta se presenta entre mujeres mayores de 30 años con un 39\% (Tabla 1) coincidiendo con la literatura observada en la Revista de obstetricia y ginecología de Venezuela, en un estudio realizado en 3070 mujeres donde se llegó a la conclusión de que el principal factor de riesgo en dicha población fue la edad mayor de 35 años. (21) De igual forma lo refiere la Revista Cubana de pediatría en su artículo, "Factores de riesgo del recién nacido macrosómico" donde Ballesté, tras realizar un estudio de 430 neonatos con peso entre 3000 a 4000 gramos, donde relacionó la edad materna superior a 35 años con la macrosomía, obtuvo resultados similares a este análisis y en estudios realizados en América Latina se concluyó que a medida que aumenta la edad materna, se incrementa el riesgo de tener un hijo macrosómico. (22)(23)

Estos resultados se asemejan a diversas publicaciones como un estudio titulado, Morbilidad y Mortalidad Neonatal Asociada a la Diabetes Gestacional, en donde el bajo peso al nacimiento representa un $21,1 \%$ de los expuestos mientras que la macrosomía en el grupo de recién nacidos hijos de madres con diabetes se encontró en el 69,3\%. (24) Lo que concuerda 
con los datos obtenidos en esta investigación que señalan que, en cuanto al peso del neonato, existe un alto porcentaje representado por un $75 \%$, con peso igual o mayor a 3999 gramos que indica ya la existencia de macrosomía fetal. Así también lo dicen Hirnle y Kowalska, es su estudio realizado en 670 gestantes, donde el $80 \%$ presentó niños macrosómicos con peso desde los 4000g lo que acierta con esta investigación.(25)Se reporta en la literatura médica que el recién nacido menor de 2500 gramos tiene mayor probabilidad de morir y se observa la disminución logarítmica a medida que aumenta el peso, pero luego de 4000 gramos se observa nuevamente un aumento de la mortalidad neonatal. (22)

En la revista chilena de ginecología y obstetricia, Garcia y Rubio(26), estudiaron a 2137 infantes macrosómicos, lograron visualizar que, a pesar de un trabajo de parto prolongado, la presentación pélvica, una desproporción céfalo pélvica y aplicación de fórceps, que son aspectos que se pueden presentar al nacimiento, el método de elección para la atención de estetipo de partos es la cesárea, ya que se considera un factor protector para dichas injurias fetales leves y graves. Pero así mismo el parto vaginal sigue siendo el destacado con un 92,3\% coincidiendo notablemente con los datos obtenidos. También lo dicen Hirnle y Kowalska(25) en donde el porcentaje de cesáreas se encuentra en un 38,5\% y partos en un $61,5 \%$ pero con el riesgo significativo de complicaciones maternas.

Wojcicki y Hessol en su análisis de 350 mujeres embarazadas con diagnóstico de diabetes gestacional, demostraron que el embarazo prolongado constituye un factor de riesgo materno

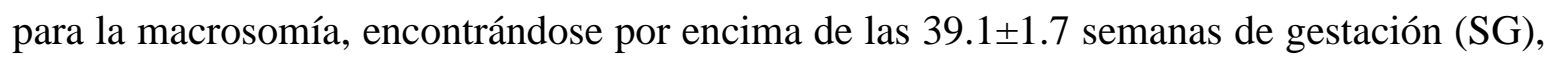
lo que se relaciona con los datos obtenidos (Tabla 4). (23) Un 58\% de neonatos se localizan dentro de las 38 a 42 SG, indicando una frecuencia muy marcada de recién nacidos a término y postérmino adecuados para la edad gestacional. Varios autores coinciden que esta variable es directamente proporcional a la incidencia de macrosomía. (27)(28).

Autores cubanos plantean que la obesidad materna y el aumento de peso durante el embarazo tienen impacto directo sobre el peso del niño al nacer y en estudios realizados por Pacora e Ingar consideraron que el IMC mayor de 25, constituyó un factor de riesgo para la macrosomía fetal, lo que se hace consistente con este estudio, ya que el peso materno entre los 71 a 80kg (IMC mayor a 30), es el que se destacó con un 32\%.(29)(30) De esta manera se ha encontrado una alta incidencia de obesidad en el grupo, y además se considera este un 
factor independiente para riesgo obstétrico, según lo refieren Campo y Posada en su estudio, "Factores de riesgo para diabetes gestacional en población obstétrica".(31)

En un trabajodo cumentado por Berle(32)se demostró que el riesgo de macrosomía es superior en las madres obesas unas tres veces comparadas con las madres normo peso y en la investigación realizada por Bergmann,(33)se reflejó como una mujer con un IMC mayor de 26 antes de la gestación y una ganancia de peso mayor a $16 \mathrm{~kg}$ durante el embarazo, tiene tendencia al nacimiento de un neonato macrosómico. Se recomienda que durante el control prenatal se debe tener especial vigilancia y una cuidadosa nutrición para evitar un incremento excesivo de peso.

Con respecto al sexo del neonato no hubo gran diferencia entre hombre y mujer existiendo predominio del primero con un 53\% y $47 \%$ respectivamente.

\section{Conclusiones.}

- En el período del 2009 al 2014 en el Hospital Vicente Corral Moscoso se encontraron 106 pacientes embarazadas con diagnóstico de Diabetes Gestacional de las cuáles, el $75 \%$ tuvieron productos con macrosomía fetal, entre las características de las madres se evidenció que la mayoría se encontraba en el grupo de edad de mayores de 30 años, con sobrepeso materno, de edad gestacional predominante fue entre las 42 o más semanas y en el $85 \%$ fue parto vaginal, por lo que se debería dar las sugerencias necesarias al personal de salud para evitar las complicaciones tanto para la madre como para el recién nacido.

\section{Recomendación}

Realizar investigaciones de tipo analítico en donde se estudien factores de riesgo.

\section{Agradecimiento}

Se expresa un gentil agradecimiento al Gerente del Hospital Vicente Corral Moscoso, Dr. Oscar Chango, por dar las facilidades brindadas para la realización de la investigación; además a la Md. Elizabeth Patiño Dra Brigith Borja, y la Dra Lorena Mosquera por su colaboración. 


\section{Referencias. Bibliográficas}

Pedersen J. La diabética gestante y su recién nacido. Problemas y tratamiento.. Scielo. 1995.

Marrero Barber MA, Plasensia Acevedo W, Idoya Gutiérrez E, Molo Amorós C, Martín Martínez A, García Hernández J. Servicio de Obstetricia y Ginecológia España: SM; 2007.

Barber M, Plasencia W, Gutierrez I, Molo C, Martín A, García J. Progresos de Obstetricia y Ginecología - Macrosomía fetal. Resultados obstétricos y neonatales Canarias : Elsevier ; 2007.

Sccuces M. Diabetes y embarazo. Revista de obstetricia y ginecología venezolana. $2011 ;:$ p. 3-12.

Huidrobo A, Prentice M, Anthony, Fullford JC. Anthropometry as predictor of gestational diabetes mellitus. Scielo. 2010;: p. 1373-1377.

Hernandez C. Macrosomía neonatal en el embarazo complicado con diabetes. [Online]; 2008. Disponible en: http://www.bvs.sld.cu/revistas/mgi/vol24_3_08/mgi06308.htm.

Castro R. Macrosomía fetal: incidencia, factores de riesgo asociados y complicaciones maternas en el hospital de ginecología y obstetricia del instituto materno infantil del estado de México, de julio 2010 a julio 2013..

Herrera E. Bioquímica Perinatal España: Ceudra; 2008.

Fascina RH, De Mucio B, Díaz Rosello L, MartÍnez G. Guias para el continuo de atencion de la mujer y el recién nacido focalizada en APS Montevideo-Uruguay: Clap; 2011.

OMS. Diabetes. [Online].; 2018.. Disponible en: http://www.who.int/mediacentre/factsheets/fs312/es/.

Salvía MD, Alvarez E, Cerqueira J. Hijo de madre diabética. [Online]; 2008. Disponible en: http://www.aeped.es/sites/default/files/documentos/15_1.pdf.

MSP. Guía práctica clínica diagnóstico y tratamiento de la diabetes en el embarazo..

Greer I. Medical problems in pregnancy. Elsevier. 2007. 
Teneumbaum K, Oron G, Chen R. Early pregnancy loss and perinatal mortality in Textbook Diabetes and Pregnancy USA; 2008.

Aguirre A. Recién nacido de peso elevado. [Online].; 2008.. Disponible en: http://www.aeped.es/sites/default/files/documentos/10_1.pdf.

Vaccaro H. Crecimiento Fetal. Revista chilena de ginecología y obstetricia. 1991;: p. 353-358.

Boulet SL, Alexander GR. Macrisomic births in the United States: : Determinants, outcomes and proposed grades of risk. Scielo. 2003.

Pacora P, Ingar W. La obesidad materna es un factor de riesgo materno-perinatal independiente San Barttolomé-Perú; 2004.

Velázquez $\mathrm{P}$, Vega $\mathrm{G}$, Martínez M. MORBILIDAD Y MORTALIDAD NEONATAL ASOCIADA A LA DIABETES GESTACIONAL. Scielo. 2010;: p. 35-41.

Chifla A. Factores de riesgo maternos y fetales en madres que padecen diabetes gestacional. Ecuador; 2013. 


\section{PARA CITAR EL ARTÍCULO INDEXADO.}

Cobos J., Rodas J. \& Carrión J., (2019). Frecuencia de Macrosomía Neonatal en mujeres embarazadas con Diabetes Gestacional en el Hospital Vicente Corral Moscoso. Cuenca Ecuador, en el periodo 2009-2014. Revista electrónica Ciencia Digital 3(2), 447-460. Recuperado desde:

http://cienciadigital.org/revistacienciadigital2/index.php/CienciaDigital/article/view/425/97 1

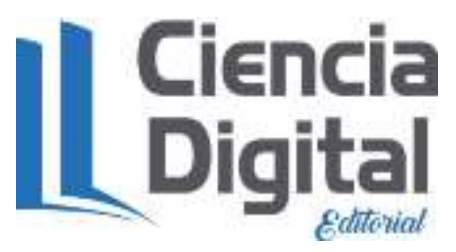

El artículo que se publica es de exclusiva responsabilidad de los autores y no necesariamente reflejan el pensamiento de la Revista Ciencia Digital.

El artículo queda en propiedad de la revista y, por tanto, su publicación parcial y/o total en otro medio tiene que ser autorizado por el director de la Revista Ciencia Digital.
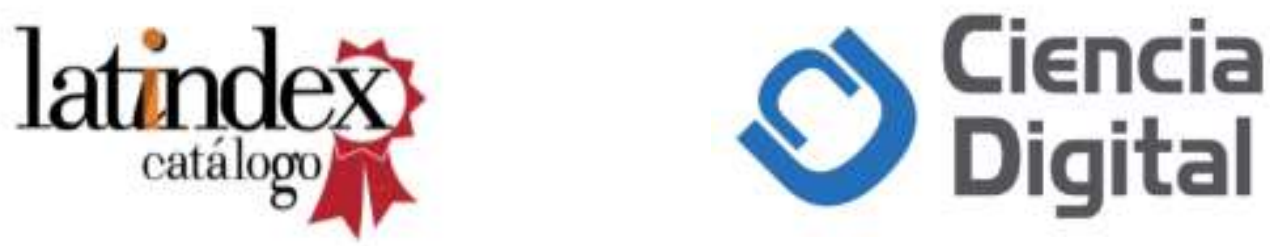